\title{
VASCULAR ENDOTHELIAL GROWTH FACTOR IS UPREGULATED IN MALIGNANT SALIVARY GLAND TUMORS
}

Felipe Paiva Fonseca - DDS, $\boldsymbol{M S} \boldsymbol{c}^{\boldsymbol{l}}$, Marcos Paulo Montanhana Basso - $\boldsymbol{D D} \boldsymbol{S}^{\boldsymbol{l}}$, Fernanda Viviane Mariano - DDS, $\boldsymbol{P h D ^ { 2 }}$, Luiz Paulo Kowalski - $\boldsymbol{M D}, \boldsymbol{P h} \boldsymbol{D}^{3}$, Márcio Ajudarte

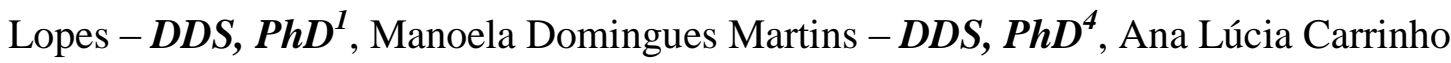
Ayroza Rangel-DDS, $\boldsymbol{P h} \boldsymbol{D}^{5}$, Ricardo Della Coletta-DDS, $\boldsymbol{P h} \boldsymbol{D}^{\mathbf{1}}$, Alan Roger Santos Silva - DDS, $\boldsymbol{P h D}^{\mathbf{l}}$ and Pablo Agustin Vargas -DDS, PhD, FRCPath ${ }^{\boldsymbol{1}}$

1. Department of Oral Diagnosis, Piracicaba Dental School, State University of Campinas - Brazil.

2. School of Medical Sciences, State University of Campinas - Brazil.

3. Department of Otorhinolaringology and Head and Neck Surgery, A.C. Camargo Cancer Center - Brazil.

4. Department of Pathology, Dental School, Federal University of Rio Grande do Sul - Brazil.

5. Department of Pathology, Dental School, State University of Western Paraná - Brazil.

\section{Corresponding author:}

\section{Prof. Dr. Pablo Agustin Vargas}

Department of Oral Diagnosis (Pathology)

Piracicaba Dental School - State University of Campinas

Limeira Street, No. 901 ZipCode:13414903 Piracicaba - São Paulo - Brazil.

Tel.: +55 1932015315 E-mail: pavargas@ fop.unicamp.br

The authors state that they have no potential conflict of interest that could bias the results obtained in this study and that the current research was supported by grants from the São Paulo State Research Foundation (Brazil), process numbers 2009/53839-2, 2012/17659-2, 2012/07519-9 and 2012/10781-7. 


\section{Abstract}

Objective: To investigate the immunoexpression of vascular endothelial growth factor (VEGF) in benign and malignant salivary gland tumors (SGT), determining its clinical and prognostic significance.

Material and methods: A total of 132 formalin-fixed, paraffin-embedded SGTs were organized in tissue microarray blocks and submitted to immunohistochemistry against VEGF protein. Slides were scanned and immunoreactions analyzed using Pixelcount V9 digital algorithm. Clinical and follow-up data were retrieved from patients' medical charts.

Results: Tumors included 50 cases of pleomorphic adenoma, 32 mucoepidermoid carcinomas, 30 adenocarcinomas not otherwise specified and 20 adenoid cystic carcinomas. A slight male preponderance was found (M:F ratio 1.1:1.0), with a mean age of 47.5 years. Parotid gland was the most affected location. VEGF expression was found in the cytoplasm of all cases analyzed with variable intensity, proving to be overexpressed in malignant tumors if compared to pleomorphic adenoma. A significant correlation of VEGF reactivity was found only with age, showing no further significant associations. Age and presence of paresthesia were the only features that predicted an inferior specific survival rate under uni and multivariate analyses. Log-rank test evidenced VEGF high expression as a potential determinant of inferior survival, although a statistical significance could not be reached.

Conclusion: Considering VEGF overexpression in malignant tumors and its potential association with an inferior survival rate, it can be speculated that this protein might be associated with SGTs pathogenesis and aggressiveness.

Keywords: Salivary gland tumors; VEGF; Vascular endothelial growth factor. 


\section{Introduction}

Salivary gland tumors (SGTs) consist of a heterogeneous group of lesions with complex clinicopathological characteristics and distinct biological behavior that correspond to approximately $3 \%$ to $10 \%$ of the neoplasms of the head and neck region. According to the World Health Organization (WHO), the global annual incidence, when all SGTs are considered, varies from 0.4 to 13.5 cases per 100,000 inhabitants (Fonseca et al., 2011).

In the last years few improvements have been made in the understanding of the molecular mechanisms involved with the development of these lesions, as a consequence chemotherapeutic protocols have not been improved significantly and surgery remains the most used therapeutic approach for patients affected by SGTs, leading to high morbidity and unsatisfactory results in many cases.

The process of new blood vessels development has been proved to be of utmost importance for neoplastic maintenance, growth and spread. A number of molecules have shown to favor angiogenic process, but vascular endothelial growth factor (VEGF) is by far the most investigated in neoplastic and non-neoplastic human diseases. VEGF is a family of growth factors that belongs to the platelet-derived growth factor (PDGF) superfamily and consists of several glycoproteins designated as VEGF-A, -B, -C, -D and -E, and the placenta growth factor (PIGF). VEGF-A, normally referred to simply as VEGF, is a dimeric glycoprotein of $34-45 \mathrm{kDa}$ that promotes angiogenesis by inducing proliferation, differentiation and migration of vascular endothelial cells (Ishak et al., 2013).

Several studies have attempted to determine the importance of VEGF for the development of different human neoplasms, including SGTs, and its utility as a prognostic factor; however, the results obtained are usually conflicting or inconclusive. 
Hence, the aim of the current study is to investigate the immunoexpression of VEGF in benign and malignant SGTs to better understand its role in the pathogenesis of these neoplasms.

\section{Material and methods}

\section{Tissue samples}

A total of 132 cases of SGTs over a 10-year period (January 2001 to December 2011) were retrieved from the archives of the Oral Diagnosis Department of the Piracicaba Dental School (Brazil), from a Southern Brazilian laboratory of surgical pathology and from the A.C. Camargo Cancer Center (Brazil). The original hematoxylineosin stained slides were reviewed by 3 oral pathologists, and the diagnoses of all cases were confirmed following the World Health Organization's 2005 Histological Typing of Salivary Gland Tumors guidelines (Barnes et al., 2005). Clinical data regarding patient's age, sex, tumor location, presence of pain and paresthesia, tumor stage, tumor recurrence and, specific and disease-free survivals were retrieved from patient's medical files. Specific survival time of each case was determined by the time difference between the date of treatment and either the date of death or last follow-up, whereas disease-free survival was obtained calculating the time between the date of treatment and the date of recurrence. Hospital records were used to determine the date of patients' last clinic visit and their status at that visit.

\section{TMA construction}

Tissue microarray (TMA) construction was done as previously described (Fonseca et al., 2014). Briefly, tumor areas were selected and marked on hematoxylineosin-stained sections using an objective marker (Nikon Corp, Tokyo, Japan). The slide 
was then overlaid on the original paraffin block to determine the corresponding area to be used. TMAs created with cases from Piracicaba Dental School and from the surgical pathology laboratory were constructed using a manual tissue arrayer (Sakura Co, Japan) and 2 representative cylindrical cores of $2.0 \mathrm{~mm}$ diameter were taken from each tissue block and then arranged sequentially into a recipient ready-to-use paraffin block (Sakura Co, Japan). Two cores of normal parotid gland and one of oral squamous cell carcinoma were inserted in the left upper corner of each recipient block for orientation. Cases retrieved from A.C. Camargo Cancer Center were arrayed as $1.0 \mathrm{~mm}$ cores also in duplicate containing one core of normal placenta tissue as internal control and for orientation. A map specifying the exact position of each case was prepared to facilitate the interpretation of the immunohistochemical results.

\section{Immunohistochemistry}

Briefly, reactions were conducted in $3-\mu \mathrm{m}$ sections of formalin-fixed, paraffinembedded tissues that were dewaxed with xylene and then hydrated in an ethanol series. Antigen retrieval was done by immersing the sections in citrate buffer solution $(\mathrm{pH}$ 6.0) for 3 minutes of pressure cooking, and the endogenous peroxidase activity was blocked using $10 \%$ hydrogen peroxide in 5 baths, each of 5 minutes. After washing in PBS buffer ( $\mathrm{pH}$ 7.4), slides were incubated overnight with primary antibody anti-VEGF (polyclonal, diluted 1:50). All slides were subsequently exposed to avidin-biotin complex and horseradish peroxidase reagents (LSAB Kit, DakoCytomation) and diaminobenzidine tetrahydrochloride (DAB, Sigma-Aldrich, St Louis, MO, USA). Finally, the slides were counterstained with Carazzi hematoxylin. Histological sections of placenta were used as positive control, whereas the negative control was obtained by omitting the specific primary antibody. 


\section{Digital analysis}

Glass slides submitted to immunohistochemistry for VEGF were scanned into high-resolution images using the Aperio Scanscope CS® Slide Scanner and the obtained digital images were analyzed with PixelCount V9 algorithm (Aperio Technologies Inc.; Vista, CA; USA). By using specific input parameters (hue value 0.0, hue width 0.0 , color saturation threshold 0.00 and intensity threshold ranging from 50 to 160 ), the percentage of cytoplasm positivity was calculated and classified in three categories according to their intensity range as weak (from 50 to 100), moderate (from 100 to 140) and strong staining (from 140 to 160). Each category received an intensity score: 1 to weak, 2 to moderate and 3 to strong staining. The final score of each tumor was calculated as the sum of the percentage of each category multiplied by their intensity scores using the following formule: $[(\%$ weak x 1$)+(\%$ moderate $\times 2)+(\%$ strong $\times 3)]$. The results always ranged from 100 to 300 .

\section{Statistical analysis}

To compare the immunoexpression of VEGF in SGTs with clinical parameters it was used Spearman correlation test. Differences in the expression of the proteins among histological subtypes were assessed by Mann-Whitney test. Log-rank test and Cox regression model were done to evaluate the prognostic significance of clinicopathological features and VEGF expression. Overall survival curve was acquired using Kaplan-Meyer method. GraphPad software (version 5.0; GraphPad Software Inc, La Jolla, CA, USA) was employed for analysis of the data. The current study was carried out in accordance with the ethical guidelines of our institution (Process number CEP/FOP 002/2013). 


\section{Results}

Clinicopathological data of the sample studied is depicted in Table 1. A small male preponderance was found $(48.5 \% v s 43.9 \%, \mathrm{M}: \mathrm{F}$ ratio $1.1: 1.0)$. The mean age of the population studied was of 47.5 years (range 8 to 91 years), whereas in the group of malignancies this mean was of 52.5 years and in the benign group of 40.4 years. Parotid

Table 1. Clinicopathological features of the sample studied.

\begin{tabular}{|c|c|c|}
\hline Parameter & $n$ & $(\%)$ \\
\hline \multicolumn{3}{|l|}{ Age } \\
\hline$<49.5$ & $57 / 132$ & 43.2 \\
\hline$\geq 49.5$ & $65 / 132$ & 49.2 \\
\hline $\bar{N} S$ & $10 / 132$ & 7.6 \\
\hline \multicolumn{3}{|l|}{ Sex } \\
\hline Male & $64 / 132$ & 48.5 \\
\hline Female & $58 / 132$ & 43.9 \\
\hline$N S$ & $10 / 132$ & 7.6 \\
\hline \multicolumn{3}{|l|}{ Site } \\
\hline Parotid & $83 / 132$ & 62.9 \\
\hline Submandibular & $17 / 132$ & 12.9 \\
\hline Sublingual & $3 / 132$ & 2.3 \\
\hline Minor glands & $18 / 132$ & 13.6 \\
\hline$N S$ & $11 / 132$ & 8.3 \\
\hline \multicolumn{3}{|l|}{ Pain* } \\
\hline Yes & $12 / 82$ & 14.6 \\
\hline No & $38 / 82$ & 46.3 \\
\hline$N S$ & $32 / 82$ & 39.1 \\
\hline \multicolumn{3}{|l|}{ Paresthesia* } \\
\hline Yes & $5 / 82$ & 6.1 \\
\hline No & $45 / 82$ & 54.9 \\
\hline$N S$ & $32 / 82$ & 39.0 \\
\hline \multicolumn{3}{|l|}{ Histologic subtype } \\
\hline Pleomorphic adenoma & $50 / 132$ & 37.9 \\
\hline MEC & $32 / 132$ & 24.2 \\
\hline Adenocarcinoma, NOS & $30 / 132$ & 22.7 \\
\hline $\mathrm{AdCC}$ & $20 / 132$ & 15.2 \\
\hline \multicolumn{3}{|l|}{ Stage* } \\
\hline $\mathrm{I} / \mathrm{II}$ & $19 / 82$ & 23.2 \\
\hline III/IV & $30 / 82$ & 36.6 \\
\hline$N S$ & $33 / 82$ & 40.2 \\
\hline \multicolumn{3}{|l|}{ Recurrence* $^{*}$} \\
\hline Yes & $14 / 82$ & 17.1 \\
\hline No & $35 / 82$ & 42.7 \\
\hline$N S$ & $33 / 82$ & 40.2 \\
\hline \multicolumn{3}{|l|}{ Follow-up } \\
\hline Alive & $25 / 82$ & 30.5 \\
\hline Dead & $26 / 82$ & 31.7 \\
\hline$N S$ & $31 / 82$ & 37.8 \\
\hline
\end{tabular}


gland was by far the most affected site ( $62.9 \%$ of the cases), followed by intra-oral minor glands (13.6\% of the cases), submandibular glands (12.9\% of the cases) and sublingual glands (2.3\% of the cases). According to histological subtypes pleomorphic adenoma accounted for $37.9 \%$ of the sample (50 cases), followed by mucoepidermoid carcinoma (24.2\% or 32 cases), adenocarcinoma NOS (22.7\% or 30 cases) and adenoid cystic carcinoma ( $15.2 \%$ or 20 cases).
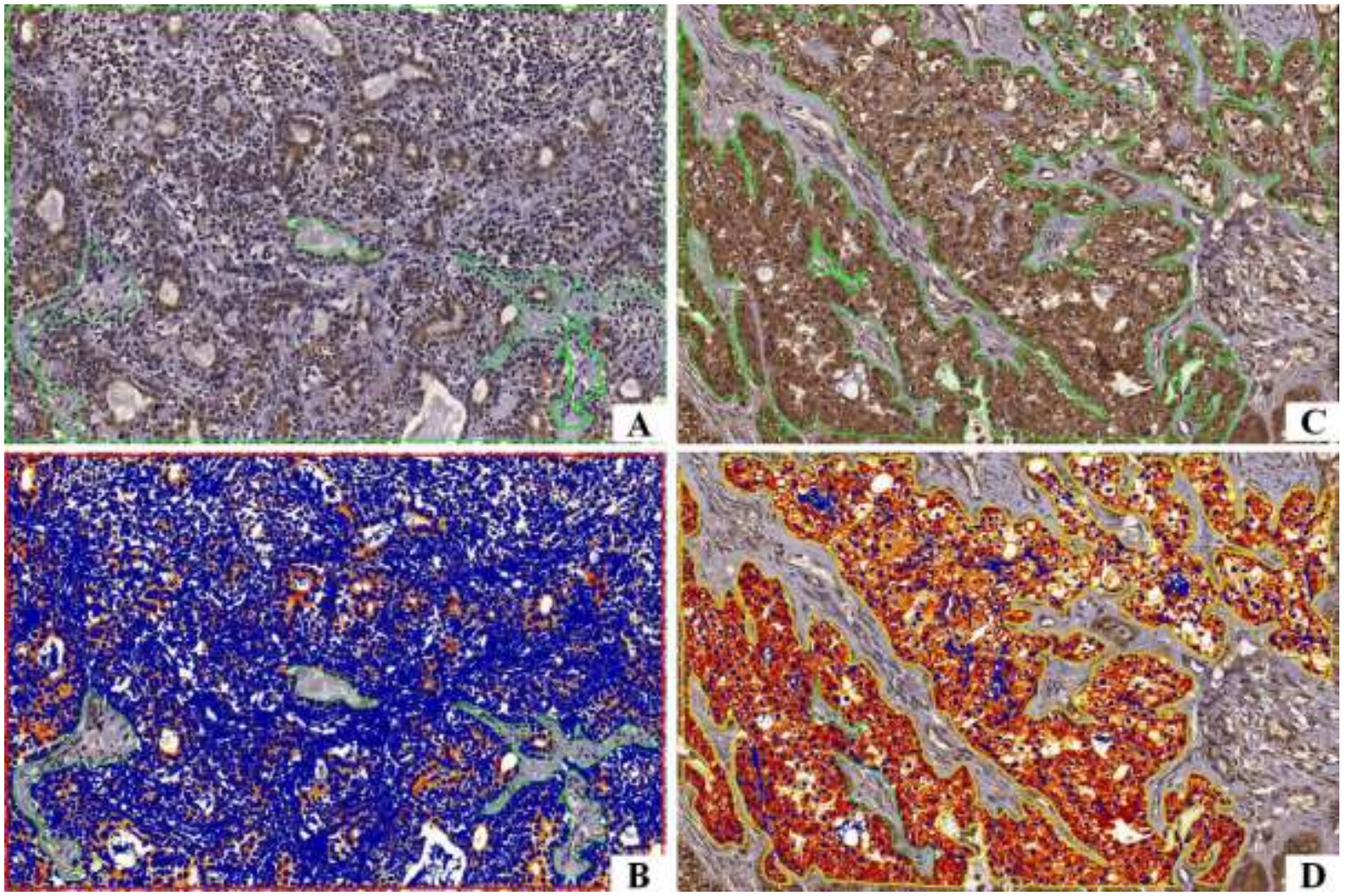

Figure 1. VEGF immunoexpression in pleomorphic adenoma and mucoepidermoid carcinoma. A) VEGF cytoplasmatic staining was significantly lower in cases of pleomorphic adenoma when compared to malignant subtypes. B) Pixelcount V9 digital analysis evidenced such low expression by an increased number of pixels categorized as blue, yellow and orange colors, representing negative, weak and moderate expression, respectively. C) Mucoepidermoid carcinoma expressed higher levels of VEGF than pleomorphic adenoma and D) digital analysis revealed a high number of pixels categorized as orange and red colors, representing moderate and strong expression. 

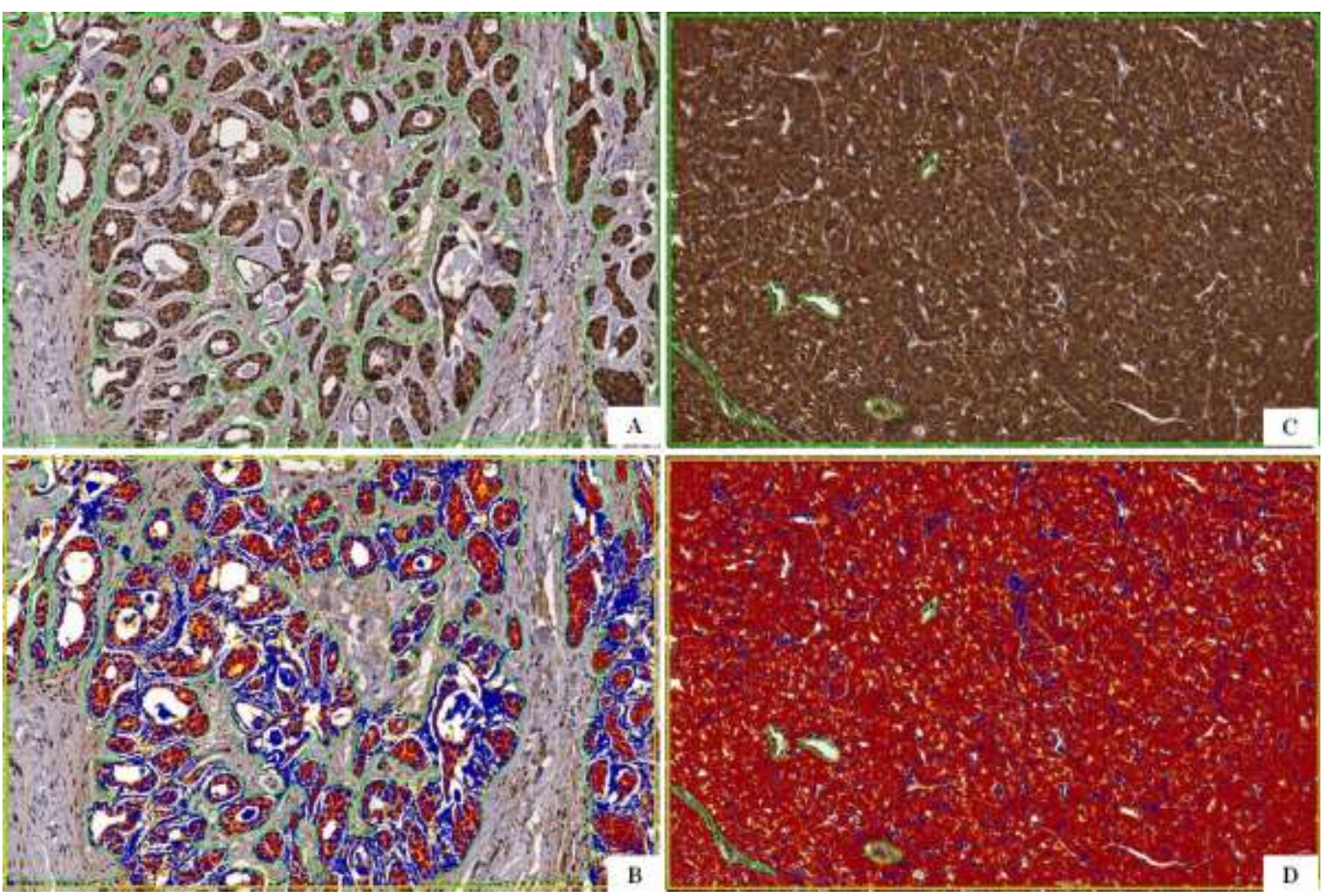

Figure 2. VEGF immunoexpression in adenoid cystic carcinoma and adenocarcinoma NOS. A) Adenoid cystic carcinoma revealed an intense reactivity for VEGF illustrated by B) digital analysis as orange and red pixels (moderate and strong expression, respectively). C) Adenocarcinoma NOS showed an even stronger expression of VEGF than MEC and ACC, but such difference was not significant. D) Performing digital analysis the algorithm identified virtually only pixels classified as red color.

VEGF expression could be identified in the cytoplasm of all cases studied with different intensities (Figures 1 and 2). A significantly increased expression was seen in malignancies than in benign pleomorphic adenoma $(\mathrm{p}<0.0001)$, whereas no significant difference was found among malignant subtypes $(\mathrm{p}>0.05)$ (Figure 3). Among malignant tumors adenocarcinoma NOS revealed the strongest expression, followed by mucoepidermoid carcinoma and adenoid cystic carcinoma. A higher VEGF expression significantly correlated with patients older than 49.5 years $(\mathrm{p}<0.0001)$, whereas no further significant correlation with other clinicopathological parameters could be found. 


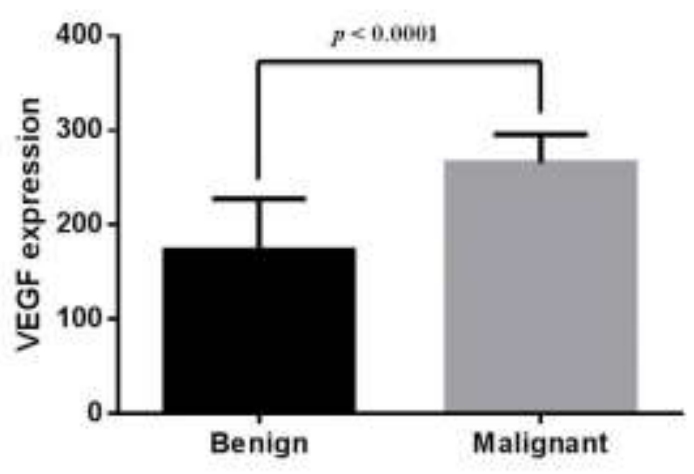

A

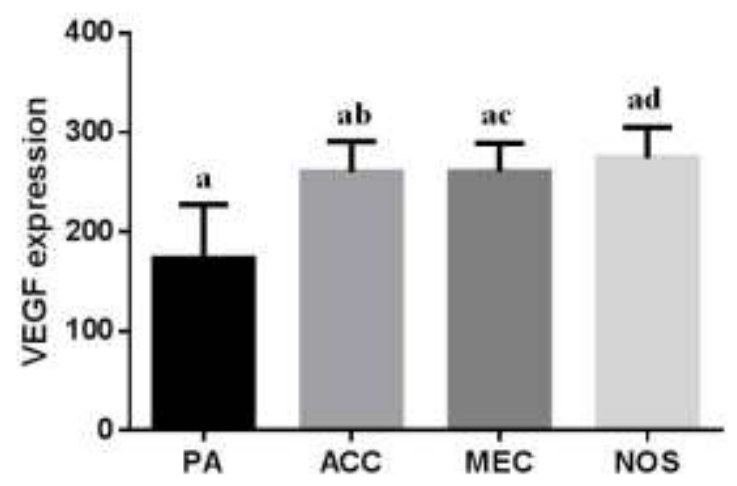

B

Figure 3. Distribution of VEGF staining between benign and malignant tumors, and among histological subtypes. A) Significant difference was obtained when VEGF expression was compared between pleomorphic adenoma and malignant tumors as a group, with an increased expression in the latter. B) By comparing the expression of VEGF among all microscopic subtypes, there was no significant difference among malignant tumors. In the graphic significant difference was found with different letters.
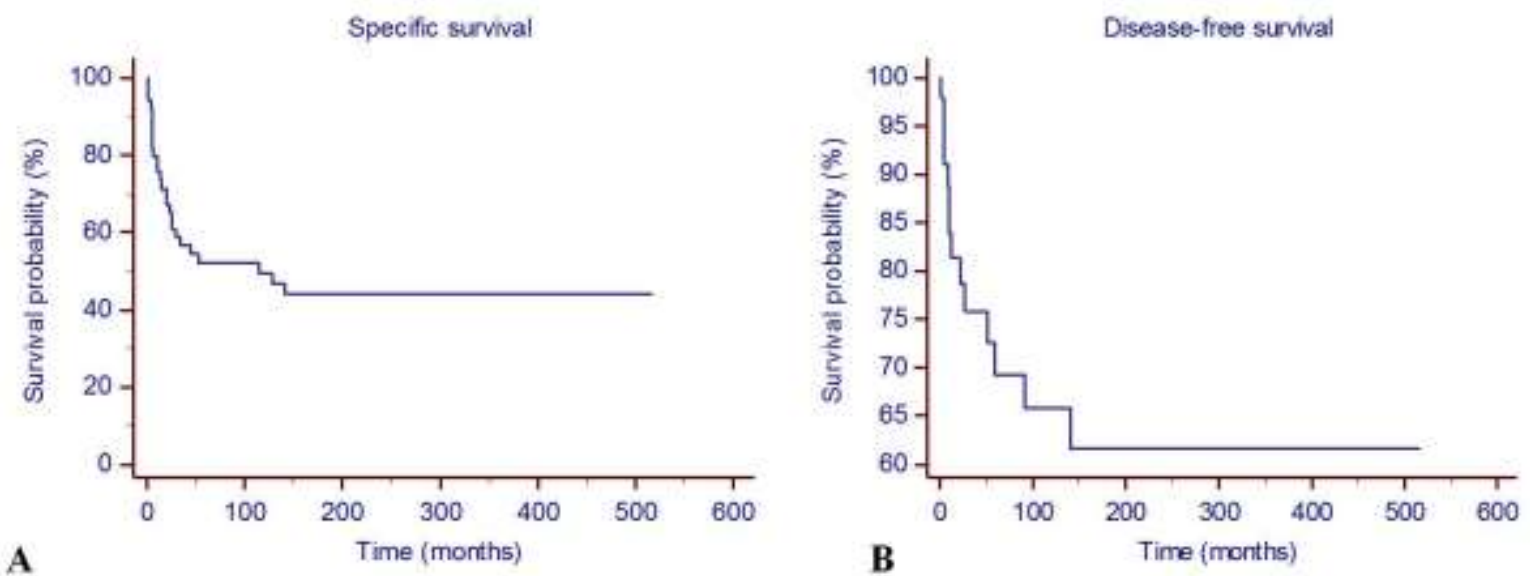

Figure 4. Kaplan-Meyer survival curves. A) Specific survival rate of the current sample was 52\%, whereas

B) disease-free survival curve reached $65 \%$.

Kaplan-Meyer survival curve demonstrated specific and disease-free survival rates of $52 \%$ and $65 \%$ for the current sample, respectively (Figure 4). Log-rank univariate analysis demonstrated that old patients and those reporting paresthesia presented a significantly inferior specific survival rate. Moreover, there was an unfavorable influence of VEGF high expression on patients' specific survival rate if 
compared to those expressing low levels of VEGF as it can be seen in Figure 5, although a statistical significance could not be reached $(p=0.1590)$. Cox regression model confirmed age and presence of paresthesia as independent predictors of a poor prognosis in this study $(p<0.05)$. Considering disease-free survival, we failed to identify any significant correlation with clinicopathological features and VEGF expression.

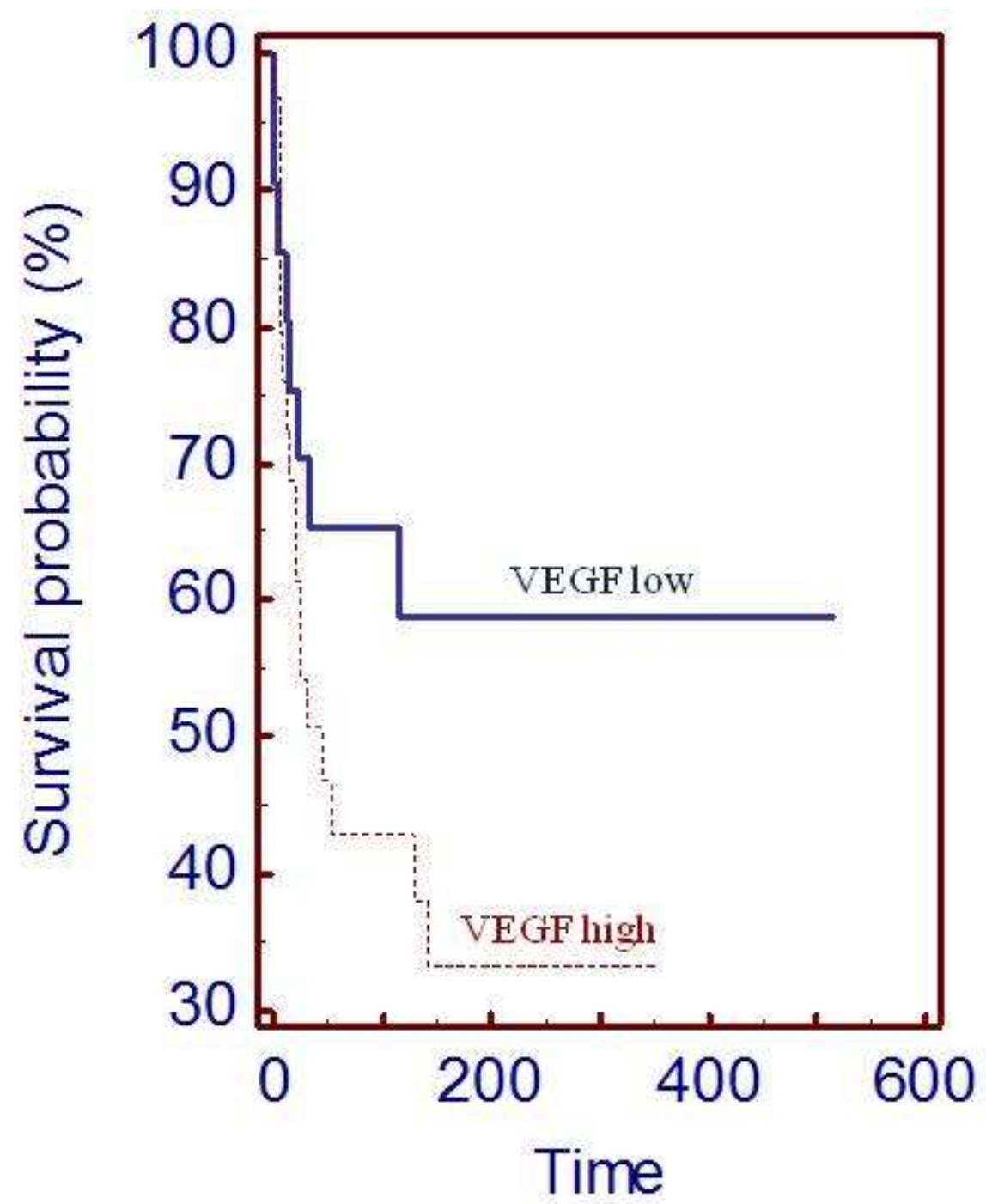

Figure 5. Survival probability calculated by univariate Log-rank test based on VEGF expression. Although the statistical significance could not be reached, a potential influence of VEGF expression on patients' survival can be easily observed. Those patients that exhibited high levels of VEGF had an inferior survival rate than those with low levels of VEGF. The lack of significance could be attributed to the relative small sample included in the present study. 


\section{Discussion}

Angiogenesis is a process tightly regulated by actions of cytokines, with the most notable being VEGF. This factor is known to exert its mitogenic activity especially on endothelial cells, initiating neoplastic angiogenic process through recruitment and proliferation of this cellular group (Zhang et al., 2009). Previous studies concluded that high levels of VEGF significantly correlate with an increased microvessel density and with an inferior survival rate of patients affected by different human neoplasms including lung, breast, colorectal, prostate, gastric and other cancers. Several authors have attempted to investigate the expression of VEGF in SGTs and correlate its presence with clinical parameters, but results remain inconclusive and divergent. In the current survey, using benign and malignant SGTs, the authors found an overexpression of VEGF in malignancies when compared to benign tumors and a potential influence in the specificsurvival rate of patients expressing high level of the protein.

VEGF family comprises members that share structural homology and includes VEGF-A (or VEGF), VEGF-B, VEGF-C, VEGF-D, VEGF-E and placental growth factor (PIGF). VEGF-A is a primary stimulator of angiogenesis, induced by hypoxia and oncogenic mutations, and is produced by a wide variety of tissues. The VEGF gene is organized into nine exons separated by seven introns. Alternative gene splicing leads to eight splice variants comprised of amino acid chains which are 121, 145, 148, 162, 165, 183, 189 and 206 amino acids in length, in addition to the more recently identified antiangiogenic VEGF165b isoform. Of these isoforms, VEGF121,VEGF165 and VEGF189 are the predominant molecular species, with the soluble, heparin-binding isoform VEGF165 being well characterized and the most often studied (Eming et al., 2006; Staton et al., 2007). VEGF activities are mediated by three high-affinity VEGF main receptors (VEGF-R1, VEGF-R2 and VEGF-R3) and two co-receptors (neuropilin-1 and neuropilin- 
2) that are expressed on both quiescent/proliferating endothelial cells and different human neoplastic cells (Staton et al., 2007). So, the prevailing concept has been that VEGF is secreted by tumor cells and its receptors are expressed by endothelial cells, increasing their proliferation and migration in a paracrine manner.

In the last years several authors aimed to investigate the expression of VEGF in SGTs. Although in the majority of the studies VEGF has been shown to be overexpressed in malignant neoplasms if compared to their benign counterparts as illustrated in the current study, and more expressed in high grade malignancies than in low grade ones, inconclusive and divergent results have been described when these authors attempted to validate VEGF as a prognostic marker. Whereas Lim et al. (2003), Zhang et al. (2005), Lequerica-Fernandez et al. (2007) and Li et al. (2010) found VEGF expression to be associated with an inferior survival rate for patients affected by different SGTs, Shi et al. (2007), Yang et al. (2010) and Lee et al. (2012) failed to demonstrate such association. In our current investigation, although we were unable to reach a statistical significance, we could identify a potential influence of VEGF high expression on the specific-survival rate of the patients, predicting an inferior survival for these patients. The lack of significance was probably consequence of the relatively small sample of malignant tumors studied. In addition, increased VEGF expression is frequently suggested to support local and distant metastases; however, Faria et al. (2011) could not confirm this biological potential of VEGF, what corroborates our findings since we failed to identify any significant association with clinical parameters.

Considering the results obtained in the present investigation and those described previously by Faria et al. (2011) and Doi et al. (1999), where VEGF is significantly more expressed in high grade malignancies and its high levels predict a poor prognosis, it can be hypothesized that this protein plays an important role in the pathogenesis of SGTs, but 
further studies are necessary to evaluate other factors and receptors like semaphorins and neuropilins, respectively, known to negatively or positively interact with VEGF to provide an increased angiogenic potential for these neoplasms.

\section{References}

1. Barnes L, Eveson JW, Reichart PA, Sidranskiy D. World Health Organization Classification of Tumours. Pathology and Genetics of Head and Neck Tumours. 1st Ed. Lyon, France: IARC Press; 2005.

2. Doi R, Kuratate I, Okamoto E, Ryoke K, Ito K. Expression of p53 oncoprotein increases intratumoral microvessel formation in human salivary gland carcinomas. $\mathrm{J}$ Oral Pathol Med 1999; 28: 259-63.

3. Eming SA, Krieg T. Molecular mechanisms of VEGF-A action during tissue repair. J Invest Dermatol Symp Proc 2006; 11(1): 79-86.

4. Faria PR, Lima RA, Dias FL, Faria PAS, Eisenberg ALA, Souza KCN, et al. Vascular endothelial growth factor and thymidine phsphorylase expression in salivary gland tumors with distinct metastatic behavior. J Oral Pathol Med 2011; 40: 456-459.

5. Fonseca FP, de Andrade BA, Rangel AL, Coletta RD, Lopes MA, de Almeida OP, et al. Tissue microarray is a reliable method for immunohistochemical analysis of pleomorphic adenoma. Oral Surg Oral Med Oral Pathol Oral Radiol 2014;117:81-88.

6. Fonseca FP, Carvalho MV, Almeida OP, Rangel ALCA, Takizawa MCH, Bueno AG, et al. Clinicopathologic analysis of 493 cases of salivary gland tumors ina Southern Brazilian population. Oral Surg Oral Med Oral Pathol Oral Radiol 2012;114:230-239. 
7. Ishak RS, Aad SA, Kyei A, Farhat FS. Cutaneous manifestations of anti-angiogenic therapy in oncology: Review with focus on VEGF inhibitors. Crit Rev Oncol Hematol 2013.

8. Lee SK, Kwon MS, Lee YS, Choi SH, Kim SY, Cho KJ, et al. Prognostic value of expression of molecular markers in adenoid cystic cancer of the salivary glands compared with lymph node metastasis: a retrospective study. World J Surg Oncol 2012.

9. Lequerica-Fernández P, Astudillo A, Vicente JC. Expression of vascular endothelial growth factor in salivary gland carcinomas correlates with lymph node metastasis. Anticancer Res 2007; 3661-3666.

10. Li H, Nong X, Chen Q, Yang Y, Li J, Li Y. Nerve growth factor and vascular endothelial growth factor: Retrospective analysis of 63 patients with salivary adenoid cystic carcinoma. Int J Oral Sci 2010; 2(1): 35-44.

11. Lim JJ, Kang S, Lee MR, Pai HK, Yoon HJ, Lee JI, et al. Expression of vascular endothelial growth factor in salivary gland carcinomas and its relations to p53, Ki67 and prognosis. J Oral Pathol Med 2003; 32: 552-561.

12. Shi L, Chen XM, Wang L, Zhang L, Chen Z. Expression of caveolin-1 in mucoepidermoid carcinoma of the salivary glands: correlation with vascular endothelial growth factor, microvessel density, and clinical outcome. Cancer 2007; 109: 1523-1531.

13. Staton CA, Kumar I, Reed MWR, Brown NJ. Neuropilins in physiological and pathological angiogenesis. J Pathol 2007; 212: 237-248.

14. Yang X, Dai J, Li T, Zhang P, Ma Q, Li Y, Zhou J, Lei D. Expression of EMMPRIN in adenoid cystic carcinoma of salivary glands: correlation with tumor progression and patients' prognosis. Oral Oncol 2010; 46: 755-60. 
15. Zhang J, Peng B, Chen X. Expressions of nuclear actor $\mathrm{kB}$, inducible nitric oxide synthase, and vascular endothelial growth factor in adenoid cystic carcinoma of salivary glands: Correlations with the angiogenesis and clinical outcome. Clin Cancer Res 2005; 11: 7334-7343.

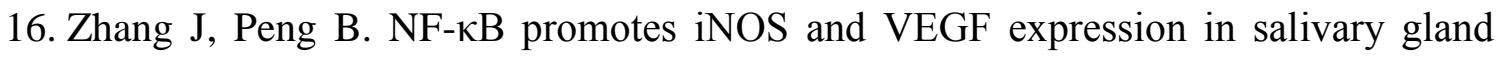
adenoid cystic carcinoma cells and enhances endothelial cell motility in vitro. Cell Prolif 2009; 42: 150-161. 